\title{
Análise das prescrições e notificações de psicotrópicos dispensadas em uma farmácia da cidade de Sobral, Ceará, Brasil
}

\section{Analysis of psychotropic prescriptions and notifications dispensed in a pharmacy of Sobral city, Ceará, Brazil}

Nícolas Matheus Ponte ${ }^{1}$. Gabriela Vieira Rolim de Sousa ${ }^{1}$. Fábio Ulisses da Silva ${ }^{1}$. George Muniz Portela da Costa $^{1}$. Maria Auxiliadora Silva Oliveira ${ }^{1}$. Danielle Rocha do Val ${ }^{1}$.

1 Centro Universitário Inta (UNINTA), Sobral, Ceará, Brasil.

\section{RESUMO}

Objetivo: Traçar o perfil das prescrições de psicotrópicos em uma farmácia comunitária do município de Sobral, Ceará. Metodologia: Foi realizada uma pesquisa de caráter quantitativo, onde foram coletadas 849 prescrições de psicotrópicos, referentes aos meses de fevereiro e março de 2016, observando os seguintes aspectos: sexo dos consumidores, tipos de psicotrópicos mais comercializados, classe terapêutica que teve maior dispensação, tipologia mais prescrita, classe médica que mais prescreveu e os possíveis erros na prescrição. Resultados: As mulheres representaram 62,6\% dos usuários; a especialidade médica que mais prescreveu foi a Clínica Geral (35,9\%), seguida pela Psiquiatria $(28,1 \%)$; os medicamentos mais comercializados foram os antidepressivos (34,5\%), seguidos pelos ansiolíticos (20,8\%), antiepilépticos (16,5\%) e antipsicóticos (12,7\%). A principal irregularidade observada foi o nome do medicamento em desacordo com a Denominação Comum Brasileira (DCB) em 73,38\% das prescrições. Conclusão: Os resultados indicam a necessidade de uma maior conscientização dos profissionais prescritores, de forma a tornar a prescrição mais correta possível, possibilitando ao usuário um atendimento pleno e eficaz.

Palavras-chave: Prescrições. Psicotrópicos. Farmácia.

\section{ABSTRACT}

Objective: To outline the profile of psychotropic prescriptions in community pharmacy in the city of Sobral, Ceará. Methodology: A quantitative research was carried out, where 849 reports of revenues were collected according to: type of prescription, therapeutic class, patient gender, medical specialty, psychotropic medication typology, and the completion of the notifications revenue by health professionals. Results: Women accounted for $62.6 \%$ of users; the medical specialty that most prescribed was the general clinic $(35.9 \%)$, followed by psychiatry $(28.1 \%)$; the most commercialized drugs were antidepressants $(34.5 \%)$, followed by anxiolytics $(20.8 \%)$, antiepileptics $(16.5 \%)$ and antipsychotics $(12.7 \%)$. The main irregularity observed was the name of the drug in disagreement with the Brazilian Common Denomination (BCD) in $73.38 \%$ of the prescriptions. Conclusion: The results indicate the need for a greater awareness of the prescribing professionals, in order to make the prescription as correct as possible, allowing the user a full and effective service.

Keywords: Prescriptions. Psychotropic drugs. Pharmacy.

Autor correspondente: Nícolas Matheus Ponte, Rua Cel. Antônio Rodrigues Magalhães, 359, Dom Expedito Lopes, Sobral, Ceará, Brasil. CEP: 62050-100. Telefone: +5588 3112-3500. E-mail: nicolasmatheus2000@hotmail.com

Conflito de interesses: Não há qualquer conflito de interesses por parte de qualquer um dos autores.

Recebido em: 01 Abr 2019; Revisado em: 30 Out 2019; Aceito em: 26 Abr 2020. 


\section{INTRODUÇÃO}

O uso de medicamento é essencial para o tratamento de diversas doenças, e a prescrição correta favorece a dispensação adequada assim como o emprego racional pelo paciente. ${ }^{1}$ Segundo a Organização Mundial da Saúde (OMS), existem seis etapas básicas para se alcançar uma terapia efetiva: definição do problema, especificação dos objetivos terapêuticos, seleção do tratamento mais eficaz e seguro, informação sobre a terapêutica e monitoração do tratamento proposto. $^{2}$

A prescrição médica é um instrumento essencial para a terapêutica e para o uso racional de medicamentos. ${ }^{1}$ De acordo com a Política Nacional de Medicamentos, a prescrição é o ato de definir o medicamento a ser consumido pelo paciente, com a respectiva posologia, expresso mediante a elaboração de uma receita. Entende-se por dispensação o ato do profissional farmacêutico de proporcionar um ou mais medicamentos ao paciente, em resposta à apresentação de uma receita elaborada por um profissional autorizado. ${ }^{3}$

Entre os modelos de receita mais comuns reconhecidos pelo Conselho Federal de Medicina (CFM), destacam-se: receita simples (medicamentos anódinos), receita de controle especial ou C (psicotrópicos, retinoides, imunossupressores, antirretrovirais e anabolizantes), receita azul ou B (psicotrópicos, como ansiolíticos) e receita amarela ou A (entorpecentes e outros psicotrópicos). ${ }^{2}$

Os fármacos psicotrópicos são definidos como aqueles que afetam o humor e o comportamento. ${ }^{4}$ São modificadores seletivos do Sistema Nervoso Central (SNC) e podem ser classificados, segundo o Sistema ATC (Anatomical Therapeutic Chemical Code), adotado pela OMS, em: anestésicos, analgésicos, antiepilépticos, antiparkinsonianos, psicolépticos (antipsicóticos, ansiolíticos, hipnóticos e sedativos) e psicoanalépticos (antidepressivos e psicoestimulantes). Devido a sua grande capacidade de afetar o organismo humano levando a dependência, esses medicamentos devem ser dispensados através de receituários de controle especial, regulamentados pela Portaria 344/98 da Secretaria de Vigilância Sanitária do Ministério da Saúde. ${ }^{5}$

Essa classe de medicamentos atua em receptores específicos que modulam a transmissão sináptica, o que pode ser percebido por meio de estímulos processados pelo sistema nervoso central. Esses processamentos ocorrem em milésimos de segundo e se repetem milhares de vezes ao longo do dia, podendo produzir diversos efeitos que variam de acordo com o tipo de neurotransmissor envolvido e o mecanismo de ação do fármaco. ${ }^{6}$

A utilização de psicotrópicos tem crescido nas últimas décadas em vários países, ocidentais e orientais. Esse uso causa impacto na sociedade, com consequências sociológicas, econômicas e sanitárias. Esse crescimento deve-se ao aumento da frequência de diagnósticos de transtornos psiquiátricos em meio à população, à introdução de novos psicofármacos no mercado farmacêutico e às novas indicações terapêuticas de psicofármacos já existentes. $^{7}$

As interações medicamentosas que podem ocorrer entre essa classe de fármacos estão cada vez maiores, devido à administração simultânea de outras drogas, bebidas e alimentos. Essas interações podem ser desejáveis ou indesejáveis. As interações desejáveis ocorrem quando os fármacos conseguem tratar as doenças concomitantemente, aumentando a eficácia e reduzindo a incidência de efeitos adversos. Enquanto as interações indesejáveis são aquelas que causam a redução da eficácia terapêutica do fármaco, podendo se obter o resultado contrário do esperado. ${ }^{8}$

O uso contínuo de medicamentos psicotrópicos pode causar dependência física e/ou psíquica, fazendo com que haja uma procura compulsiva pelo fármaco, desenvolvendo o vício, o que prejudica o comportamento social do indivíduo. Poderá também causar degeneração de determinados tipos celulares, caso haja automedicação em doses excessivas, acarretando lesões irreversíveis. ${ }^{9}$

Diante da seriedade da manipulação e comercialização das substâncias psicotrópicas, este trabalho tem como objetivo traçar o perfil das prescrições de psicotrópicos dispensadas em uma farmácia comunitária do município de Sobral, e avaliar os possíveis erros que possam estar presentes.

\section{MATERIAIS E MÉTODOS}

Trata-se de um estudo descritivo, retrospectivo e exploratório, com abordagem quantitativa, realizado em uma farmácia comunitária privada do município de Sobral, CE. Foi feita uma solicitação à direção geral da farmácia para autorizar o acesso à amostra estudada. Os critérios de inclusão utilizados foram prescrições de psicotrópicos (tipos B e C) dispensadas no período de fevereiro e março de 2016. Foram excluídas da pesquisa as prescrições de outras classes farmacêuticas que não psicotrópicos, e aquelas que não corresponderam ao período supracitado. A identificação do paciente e do prescritor foi mantida em sigilo.

As prescrições foram analisadas por um estudante de medicina sob auxílio e supervisão do farmacêutico responsável. Para a coleta dos dados foi utilizada uma planilha impressa confeccionada em Excel, constando as variáveis analisadas: o sexo dos consumidores, os tipos de psicotrópicos mais comercializados (B, C1 e C2), a classe terapêutica que teve maior dispensação, a tipologia farmacêutica (medicamento genérico, similar ou de referência), a especialidade médica que mais prescreveu e os possíveis erros na prescrição.

Os resultados foram registrados, considerando as informações e suas especificidades, em tabelas, visto que a pesquisa foi quantitativa, e os dados consolidados foram compilados nos programas Microsoft Office Word e Excel 2013. Os resultados foram expressos como frequência absoluta $(\mathrm{N})$ e frequência relativa $(\%)$. 
O projeto foi cadastrado na Plataforma Brasil e submetido ao Comitê de Ética em Pesquisas envolvendo seres humanos (CEP) da Universidade Estadual do Vale do Acaraú, juntamente com a Carta de Anuência, tendo sido aprovado com o número de parecer 2.054.257 em 09 de Maio de 2017. A pesquisa foi realizada de acordo com os critérios estabelecidos pela Declaração de Helsinki com as suas modificações.

\section{RESULTADOS}

Os dados apresentados são referentes ao levantamento ocorrido nos meses de fevereiro e março de 2016, onde foram analisadas 849 receitas e notificações de receitas correspondentes à dispensação de medicamentos psicotrópicos, sendo que destas, as mais prescritas foram receituários de controle especial em duas vias (C1), com 73,7\% do total, conforme a Tabela 1. Não foram identificados receituários do tipo B2.

Quanto ao gênero dos usuários, observou-se uma prevalência do sexo feminino em $62,7 \%$ notificações, enquanto o sexo masculino totalizou $37,3 \%$, como pode ser observado na Tabela 2.

Com relação ao tipo de medicamento mais comercializado na farmácia do estudo em questão (Tabela 3), evidenciou-se uma maior dispensação de medicamentos de referência, equivalente a $37,8 \%$ das prescrições avaliadas, seguida por $35,6 \%$ de prescrições de similares, e $26,6 \%$ de medicamentos genéricos.

Tabela 1. Distribuição dos tipos de notificações de receita de uma farmácia em Sobral-CE no período de fevereiro e março de 2016.

\begin{tabular}{lll}
\hline Tipos de receitas & $\mathbf{N}$ & $\mathbf{\%}$ \\
\hline Tipo B1 & 177 & $20,85 \%$ \\
Tipo C1 & 626 & $73,73 \%$ \\
Tipo C2 & 46 & $05,42 \%$ \\
\hline
\end{tabular}

Tabela 2. Distribuição do sexo dos consumidores de psicotrópicos analisados em receitas dispensadas em uma farmácia em Sobral-CE no período de fevereiro e março de 2016.

\begin{tabular}{lll}
\hline Sexo & N & \% \\
\hline Feminino & 532 & $62,66 \%$ \\
Masculino & 317 & $37,34 \%$ \\
\hline
\end{tabular}

Tabela 3. Distribuição dos tipos de medicamentos comercializados na farmácia em estudo durante o período de fevereiro e março de 2016.

\begin{tabular}{lll}
\hline Tipos de medicamento & $\mathbf{N}$ & $\mathbf{\%}$ \\
\hline Medicamentos de referência & 321 & $37,81 \%$ \\
Medicamentos similares & 302 & $35,57 \%$ \\
Medicamentos genéricos & 226 & $26,62 \%$ \\
\hline
\end{tabular}

Quanto aos prescritores, a especialidade mais frequentemente associada a prescrição de psicotrópicos foi a Clínica Geral, representando $35,9 \%$ do total, seguida pela Psiquiatria $(28,1 \%)$ e pela Neurologia $(11,7 \%)$ (Tabela 4$)$.

No que se refere à classe farmacológica (Tabela 5), os fármacos antidepressivos foram os mais comercializados, totalizando $34,5 \%$ das prescrições, seguido pelos ansiolíticos (20,8\%), antiepilépticos $(16,5 \%)$ e antipsicóticos $(12,7 \%)$.

Com relação ao cumprimento da legislação sanitária, a principal irregularidade constatada foi o nome do medicamento em desacordo com a Denominação Comum Brasileira (DCB), em 73,4\% das prescrições. A ilegibilidade (1,4\%), ausência de dados na identificação do usuário $(0,2 \%)$ e ausência de dados na identificação do emitente $(0,1 \%)$ também foram erros encontrados na presente pesquisa, conforme Tabela 6 .

Tabela 4. Distribuição das especialidades médicas identificadas nas receitas de controle especial em uma farmácia no município de Sobral-CE durante o período de fevereiro e março de 2016.

\begin{tabular}{lll}
\hline Especialidade & $\mathbf{N}$ & $\mathbf{\%}$ \\
\hline Clínica Geral & 305 & $35,92 \%$ \\
Psiquiatria & 239 & $28,15 \%$ \\
Neurologia & 99 & $11,66 \%$ \\
Dermatologia & 53 & $06,24 \%$ \\
Outras* & 153 & $18,02 \%$ \\
\hline
\end{tabular}

*Cardiologia, pediatria, geriatria, ginecologia, ortopedia, medicina da família.

Tabela 5. Distribuição das classes farmacológicas de psicotrópicos comercializados em uma farmácia no município de Sobral-CE durante o período de fevereiro e março de 2016.

\begin{tabular}{lll}
\hline Classe farmacológica & $\mathbf{N}$ & $\mathbf{\%}$ \\
\hline Antidepressivos & 293 & $34,51 \%$ \\
Ansiolíticos & 177 & $20,85 \%$ \\
Antiepiléticos & 140 & $16,49 \%$ \\
Antipsicóticos & 108 & $12,72 \%$ \\
Outros* & 131 & $15,43 \%$ \\
\hline
\end{tabular}

*Hipnóticos, anti-Parkinson, anti-Alzheimer, estabilizadores do humor, retinoides

Tabela 6. Irregularidades no preenchimento de prescrições dispensadas em uma farmácia no município de Sobral-CE durante o período de fevereiro e março de 2016.

\begin{tabular}{lll}
\hline Irregularidades & $\mathbf{N}$ & $\mathbf{\%}$ \\
\hline Nome em desacordo com a DCB & 623 & $73,38 \%$ \\
Ilegibilidade & 12 & $1,41 \%$ \\
Ausência de dados na identificação do usuário & 02 & $0,23 \%$ \\
Ausência de dados na identificação do emitente & 01 & $0,12 \%$ \\
\hline
\end{tabular}




\section{DISCUSSÃO}

O receituário de controle especial $(\mathrm{C} 1)$ é utilizado para a prescrição de medicamentos de tarja vermelha, como retinoides de uso tópico, imunossupressores e antirretrovirais, anabolizantes e antidepressivos. A notificação de receita do tipo B é um impresso de cor azul utilizado para a prescrição de medicamentos de tarja preta, principalmente ansiolíticos. A receita do tipo $\mathrm{C} 2$ é reservada para a prescrição de retinoides de uso sistêmico, com destaque para a isotretinoína. ${ }^{2}$

O consumo de medicamentos pelo sexo feminino tem maior prevalência, possivelmente pelo fato das mulheres apresentarem maior preocupação com a saúde, sendo mais conscientes com questões relacionadas ao autocuidado, pois tendem a utilizar com maior frequência os serviços de saúde, portanto, elas são mais familiarizadas com a aderência aos tratamentos farmacológicos. ${ }^{10}$

Ademais, no estudo realizado por Torres et al. (2014) em uma farmácia do Maranhão, afirmou-se que as mulheres são mais afetadas por doenças psiquiátricas. ${ }^{11}$ Segundo Sadock (2008), a prevalência de transtornos depressivos é cerca de duas vezes maior nas mulheres, podendo-se supor que as razões para isso envolvam diferenças hormonais, efeitos estressores diferentes para os sexos e modelos comportamentais. ${ }^{12}$

A prevalência da compra de medicamentos de referência, por mais que a maioria possua um maior custo em relação aos similares e aos genéricos, se dá provavelmente devido a influência das empresas farmacêuticas no âmbito social e ao descumprimento da legislação vigente. A OMS preconiza que $100 \%$ das prescrições devem ser feitas pelo nome genérico, o que implica na necessidade de conscientização por parte dos profissionais de saúde. ${ }^{1}$

O medicamento genérico é equivalente a um produto de referência, que se pretende ser com este intercambiável, geralmente produzido após a expiração ou renúncia da patente ou de outros direitos de exclusividade, com comprovada eficácia, segurança e qualidade, e designado pela Denominação Comum Brasileira (DCB) ou, na sua ausência, pela Denominação Comum Internacional (DCI). Tem como base legal a Lei ${ }^{\circ}$ 9.787/99 e objetiva estimular a variedade de oferta no mercado, possibilitando uma melhora da sua qualidade e redução do preço, facilitando o acesso da população aos tratamentos. ${ }^{13}$

Além de ser de uso obrigatório por lei no serviço público brasileiro (Sistema Único de Saúde - SUS), a denominação genérica também reduz as confusões entre nomes de medicamentos e facilita a obtenção de informações a respeito de fármacos em literatura internacional, já que a denominação comercial varia conforme o país. ${ }^{14}$

Segundo a Organização Pan-Americana de Saúde (OPAS), a oferta cada vez maior de medicamentos similares, equivalentes e alternativas farmacêuticas, associada ao intenso trabalho de marketing, estimula a prescrição e o uso irracional de fármacos. ${ }^{15}$
No que se refere à especialidade do prescritor, os resultados aqui apresentados foram compatíveis com os de Costa e Oliveira (2017), onde constatou-se que o clínico geral foi o profissional que mais emitiu receitas de psicotrópicos, correspondendo a $38,8 \%$ das prescrições analisadas, seguido pelas especialidades de Psiquiatria (19,4\%) e Dermatologia $(13,0 \%) .{ }^{16}$

Segundo Ferrari et al. (2013), esperava-se que psiquiatras e neurologistas tivessem um perfil de prescrição diferenciado, uma vez que esses especialistas conhecem profundamente as propriedades farmacológicas dos psicotrópicos e os riscos inerentes a sua utilização. Clínicos gerais, por sua vez, têm menos conhecimento acerca dos efeitos clínicos e adversos desses medicamentos, prescrevendo-os amplamente. ${ }^{17}$

Segundo Mari e Jorge (2018), o clínico geral (ou o médico generalista) é o profissional mais atuante em saúde mental no Brasil, fenômeno também observado em países como a Inglaterra, os Estados Unidos e o Canadá. A estimativa é de que apenas 1 a cada 20 casos psiquiátricos presentes na Clínica Geral seja encaminhado para o médico especialista. Isso poderia refletir, em parte, o consumo indiscriminado destes fármacos de controle especial. ${ }^{18}$

Em relação à classe farmacológica, os resultados corroboram com o estudo de Assini e Back (2017), realizado em farmácias do município de Monte Carlo (SC), no qual foi constatado que os antidepressivos estiveram presentes na maioria das prescrições médicas, sendo o número de casos equivalente a $34,5 \%$ do total, seguidos pelos benzodiazepínicos $(23,4 \%)$ e anticonvulsivantes $(12,2 \%)$. Os principais representantes dessas classes foram a amitriptilina, o clonazepam e a carbamazepina, respectivamente. ${ }^{7}$

O grande índice encontrado para a comercialização de antidepressivos no presente estudo relaciona-se com o crescimento do diagnóstico de transtornos depressivos, ao surgimento de novos fármacos e à ampliação das indicações terapêuticas desses medicamentos. ${ }^{19}$

Os ansiolíticos estão entre os fármacos mais consumidos no mundo devido ao seu notório efeito no tratamento, ocupando o segundo lugar. Os benzodiazepínicos representam os medicamentos de escolha para o tratamento de ansiedade aguda e agitação. ${ }^{1}$ Diante do risco da dependência psicológica, a utilização em longo prazo desses fármacos deve ser monitorada cuidadosamente. ${ }^{12}$

São considerados erros de prescrição quando há equívocos referentes à decisão ou redação, não intencionais, que podem diminuir a efetividade do tratamento. ${ }^{1}$ Os erros oriundos das prescrições médicas podem ainda acarretar graves danos à saúde do paciente, sendo, portanto, indispensável a implementação de estratégias de identificação e prevenção. ${ }^{20}$ A presença de erros em prescrições é frequente, indicando a necessidade de qualificação dos prescritores e a atuação direta do farmacêutico no suporte a decisões clínicas e na oferta de informações e orientações sobre os medicamentos, visando a redução de erros potencialmente prejudiciais aos usuários e a promoção de uma terapia medicamentosa segura e eficaz. ${ }^{21}$ 
Quanto às irregularidades encontradas, o resultado foi semelhante ao estudo de Firmo et al. (2013), realizado em um município do Maranhão, o que demonstra a forte influência da indústria farmacêutica e o descumprimento, por parte dos médicos do setor público (SUS), em relação à legislação que obriga a prescrição medicamentosa segundo a DCB. ${ }^{1}$ A não adesão à DCB pode comprometer o acesso aos medicamentos, pois o paciente fica sem a opção da escolha de um medicamento mais barato com mesmo perfil de segurança, qualidade e eficácia. $^{22}$

Por fim, a presente pesquisa mostrou ainda que existe a necessidade, por parte dos prescritores, quanto ao relato de informações primordiais para a dispensação correta do medicamento, assim como o uso pelo paciente, resultando em uma terapêutica ineficaz. ${ }^{1}$

\section{CONCLUSÃO}

O presente estudo apresentou resultados relevantes no tocante à dispensação de fármacos psicoativos em uma farmácia

\section{REFERÊNCIAS}

1. Firmo WC, Paredes AO, Cunha CL, Torres AG, Buccini DF. Análise das prescrições médicas de psicotrópicos de uma farmácia comercial no município de Bacabal, Maranhão. J Manag Prim Health Care. 2013;4(1):10-18.

2. Madruga CM, Souza ES. Manual de orientações básicas para prescrição médica. João Pessoa: Ideia; 2009.

3. Brasil. Ministério da Saúde. Política Nacional de Medicamentos. Brasil: Ministério da Saúde; 2001.

4. Rang HP, Dale MM, Ritter JM, Flower RJ. Farmacologia. 6. ed. Rio de Janeiro: Elsevier; 2007.

5. Brasil. Ministério da Saúde. Portaria n 344, de 12 de maio de 1998. Aprova o regulamento técnico sobre substâncias e medicamentos sujeitos a controle especial. Brasília: Ministério da Saúde; 1998.

6. Canesin R, Machado JA, Oliveira AC, Antônio NC, Rocha JR, Biazotto G. Psicotrópicos: revisão de literatura. Revista Científica Eletrônica de Medicina Veterinária. 2008;6(11):41-7.

7. Assini FL, Back JT. Análise das prescrições de psicotrópicos em farmácias privadas na cidade de Monte Carlo, Santa Catarina. Rev Eletr Farm. 2017;14(2):5-14.

8. Ribeiro DF, Lima CE. Possíveis interações farmacológicas entre psicotrópicos e a politerapia realizada por pacientes adultos da cidade de Anápolis, Goiás [monografia]. Anápolis: Universidade Estadual de Goiás; 2011. 100 p.

9. Silva DM. Avaliação do consumo de medicamentos psicotrópicos no município de Pacatuba [monografia]. Fortaleza: Escola de Saúde Pública do Estado do Ceará; 2009 [acesso em: 15 ago 2018]. Disponível em: https://docplayer.com.br/19774799-Avaliacao-do-consumo-demedicamentos-psicotropicos-no-municipio-de-pacatuba.html

10. Ignácio VT, Nardi HC. A medicalização como estratégia biopolítica: um estudo sobre o consumo de psicofármacos no de médio porte do município de Sobral (CE), tendo como base o perfil das prescrições e erros presentes. Não houve discrepâncias no que se concerne ao perfil dos consumidores, prescritores e aos fármacos mais dispensados por meio de prescrições de receituário especial, quando comparado a estudos prévios com a mesma temática.

Quanto ao preenchimento das prescrições, a principal irregularidade encontrada foi o descumprimento da legislação que obriga a prescrição medicamentosa segundo a DCB, especialmente no âmbito do sistema público de saúde brasileiro. Portanto, faz-se necessária uma maior conscientização dos prescritores, de forma a tornar a prescrição mais correta possível, possibilitando ao usuário um atendimento pleno e eficaz.

Ademais, há a necessidade de aprofundar o perfil dos usuários de psicotrópicos, acerca das condições socioeconômicas, nível de escolaridade e presença de comorbidades, para melhor compreensão da realidade do município e, assim, promover o uso racional desses medicamentos.

contexto de um pequeno município do Rio Grande do Sul. Psicol Soc. 2007;19(3):88-95.

11. Torres ML, Sousa LM, Melo GC, Magalhães AA Júnior, Firmo WC. Prescrição de psicotrópicos e especialidade médica: estudo em uma farmácia comercial no município do Maranhão. Revista Científica do ITPAC, Araguaína. 2014;7(4):[6 p.].

12. Sadock BJ, Sadock VA. Manual conciso de psiquiatria clínica. 2. ed. Porto Alegre: Artmed; 2008.

13. Brasil. Lei $n^{\circ} 9.787$, de 10 de fevereiro de 1999. Altera a lei $n^{\circ}$ 6.360 , de 23 de setembro de 1976, que dispõe sobre a vigilância sanitária, estabelece o medicamento genérico, dispõe sobre a utilização de nomes genéricos em produtos farmacêuticos e dá outras providências. Brasília: [publisher unknown]; 1999 [acesso em: 05 jul 2018]. Disponível em: http://www.planalto.gov.br/ccivil_03/Leis/ L9787.htm.

14. Wannmacher L, Ferreira MB. Normatização da prescrição medicamentosa. In: Fuchs FD, Wannmacher L (eds.). Farmacologia clínica: fundamentos da terapêtica racional. 2. ed. Rio de Janeiro: Guanabara Koogan; 1998. p. 54-7.

15. Marin N, Luiza VL, Castro CG, Santos SM. Assistência farmacêutica para gerentes municipais. Rio de Janeiro: OPAS/OMS; 2003.

16. Costa GM, Oliveira MA. Estudo das prescrições de psicotrópicos em uma farmácia da cidade de Sobral, Ceará, Brasil. Infarma. 2017;29(1):27-33.

17. Ferrari CK, Brito LF, Oliveira CC, Moraes EV, Toledo OR, David FL. Falhas na prescrição e dispensação de medicamentos psicotrópicos: um problema de saúde pública. Rev Ciênc Farm Básica Apl. 2013;34(1):109-16.

18. Mari JJ, Jorge MR. Transtornos psiquiátricos na clínica geral. Psychiatry on line Brazil [Internet]. 1997;2(5):[acesso em: 22 set 
2018]. Disponível em: http://www.polbr.med.br/ano97/tpqcm.php.

19. Rodrigues MA, Facchini LA, Lima MS. Modificações nos padrões de consumo de psicofármacos em localidade do Sul do Brasil. Rev Saúde Pública. 2006;40(1):107-14.

20. Lima TA, Gouveia MI, Pereira LL, Godoy MF. Erros de prescrições médicas em drogaria. Infarma. 2016;28(1):16-21.
21. Bandeira VA, Hermann CT, Siqueira CM, Oliveira KR. Análise das prescrições dispensadas em uma unidade básica de saúde do município de Ijuí - RS. Saúde (Santa Maria). 2015;41(1):229-38.

22. Lemos GS, Lemos LB, Cardoso RA, Nascimento LE, Mota IV. Medicamentos sob controle especial: uma análise dos erros de medicação e indicadores de prescrição. Revista Eletrônica de Farmácia. 2016;8(1):45-54.

\section{Como citar:}

Ponte NM, Sousa GV, Silva FU, Costa GM, Oliveira MA, Val DR. Análise das prescrições e notificações de psicotrópicos dispensadas em uma farmácia da cidade de Sobral, Ceará, Brasil. Rev Med UFC. 2020 out-dez;60(4):5-10. 